\title{
Experimental results from CMS
}

\author{
John Strologas ${ }^{1}$ (for the CMS collaboration) \\ ${ }^{1}$ Department of Physics, University of Ioannina, Ioannina 45110, Greece
}

\begin{abstract}
We present some of the latest results of the CMS experiment, covering analyses in QCD multijet, top, bottom, Higgs, forward/small- $x$ QCD, heavyion, exotic, and supersymmetry physics utilizing LHC integrated luminosity up to $\sim 80 \mathrm{fb}^{-1}$.
\end{abstract}

\section{Introduction}

CMS, as a major general-purpose detector at the LHC, provides an abundance of high-quality data that enable the performance of analyses on a plethora of physics topics. We present some of the legacy and most recent results with some emphasis on Quantum Chromodynamics (QCD) relevance.

\section{QCD multijet physics}

Double-differential inclusive jet production cross sections have been measured with $8 \mathrm{TeV}$ [1] and 13-TeV [2] data, corresponding to luminosity of $19.7 \mathrm{fb}^{-1}$ and $71 \mathrm{pb}^{-1}$ respectively. Particle-flow [3] jets are reconstructed with the anti- $k_{t}$ algorithm [4] with $R$ distance parameter of 0.4 and 0.7. Results demonstrate excellent understanding of QCD next-to-leading order (NLO) fixed-order calculations corrected for non-perturbative (NP) effects from parton showering, hadronization and multiparton interactions. As shown in Figs. 1 and 2, the Standard Model (SM) agrees with the observed distributions for a cross-section range of 18 orders of magnitude, up to $2 \mathrm{TeV}$ in jet-transverse momentum $p_{T}$ and in seven absolute rapidity $y$ bins up to 4.7 , for jets reconstructed with both $R=0.4$ and $R=0.7$.

Studies of azimuthal correlations between the leading two jets or any pair of jets [5] compare 35.9- $\mathrm{fb}^{-1}, 13-\mathrm{TeV}$ data to event generators at LO (Pythia8, Herwig++, MadGRAPH+Pythia8) and NLO (Powheg, Herwig7). Levels of agreement depend on jet multiplicity.

The triple-differential dijet cross section, measured as a function of $p_{T}$, the difference, and sum of jet rapidities with $19.7-\mathrm{fb}^{-1}, 8-\mathrm{TeV}$ data [6], is not described sufficiently by NLO QCD calculations with NP and electroweak corrections. This fact is used for constraining the proton parton-distribution functions (PDF). The inclusion of these CMS results in the PDF fits changes the gluon PDF shape at lower $x$ values and reduces the systematic uncertainty effect at higher $x$, compared to deep-inelastic-scattering-only fits, as shown in Fig. 3.

A measurement of the ratio of inclusive 3 -jet to 2 -jet cross section with $R=0.7$ jets with $19.7-\mathrm{fb}^{-1}$ data from $8-\mathrm{TeV}$ collisions provides an accurate measurement of the strong coupling constant $a_{s}$ [7], due to canceling common systematic uncertainties on the numerator 

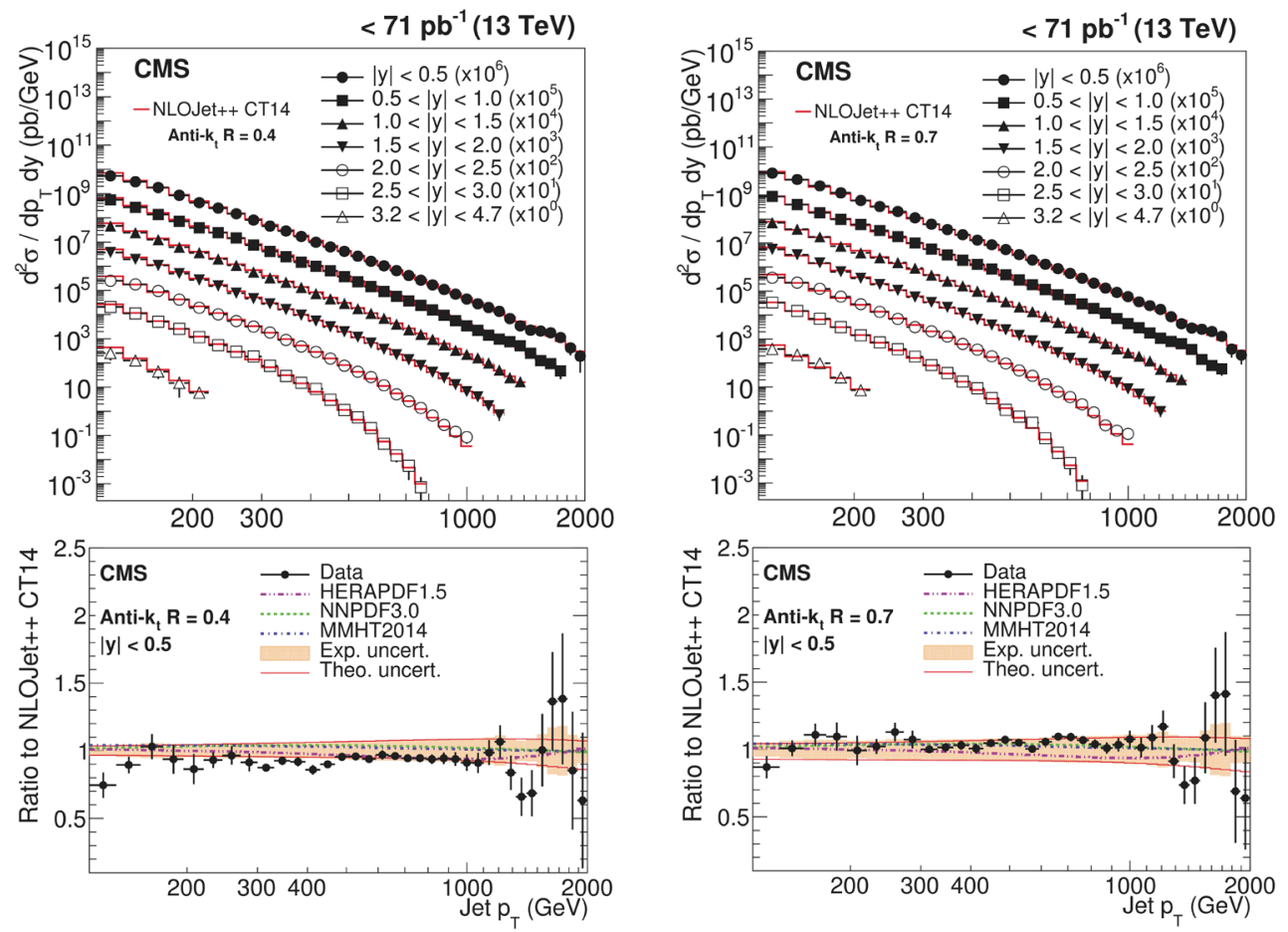

Figure 1. Upper plot: Double-differential inclusive jet cross section for $R=0.4$ jets at $13 \mathrm{TeV}$. Lower plot: comparison with NLO-in-QCD theoretical prediction, with NP and electroweak corrections, for $|y|<0.5$.
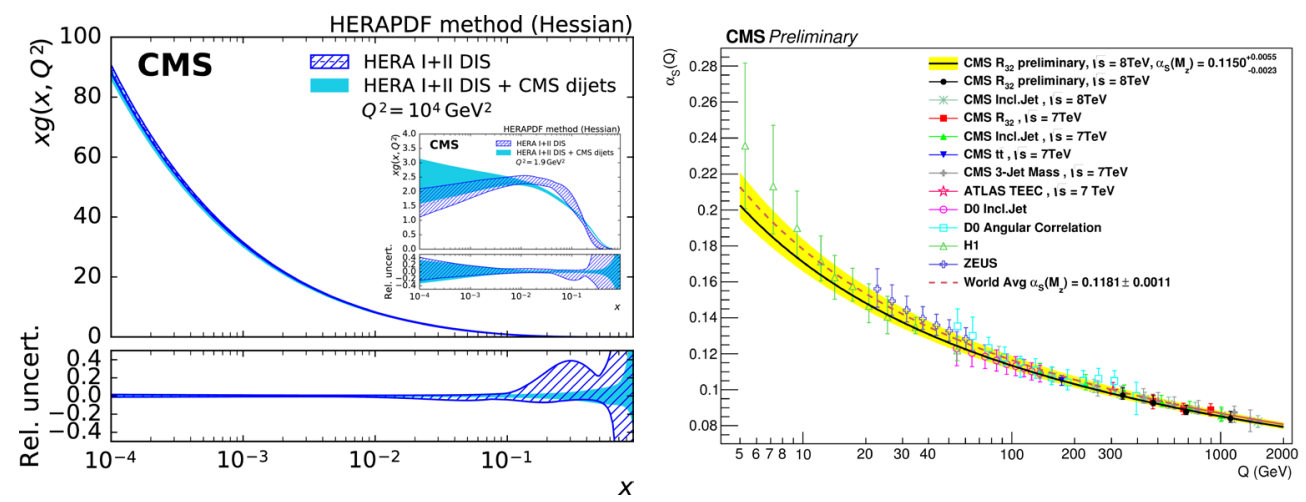

Figure 3. Extraction of the gluon PDF with inclusion of input from the CMS triple-differential inclusive jet cross section at $Q^{2}=10^{4} \mathrm{GeV}^{2}$ (insert: $Q^{2}=1.9 \mathrm{GeV}^{2}$ ).

Figure 2. Upper plot: Double-differential inclusive jet cross section for $R=0.7$ jets at $13 \mathrm{TeV}$. Lower plot: comparison with NLO-in-QCD theoretical prediction, with NP and electroweak corrections, for $|y|<0.5$.

Figure 4. Extraction of the $\alpha_{S}$ from the CMS $R_{32}$ analysis (black data points) and comparison with world average and other experimental measurements. 

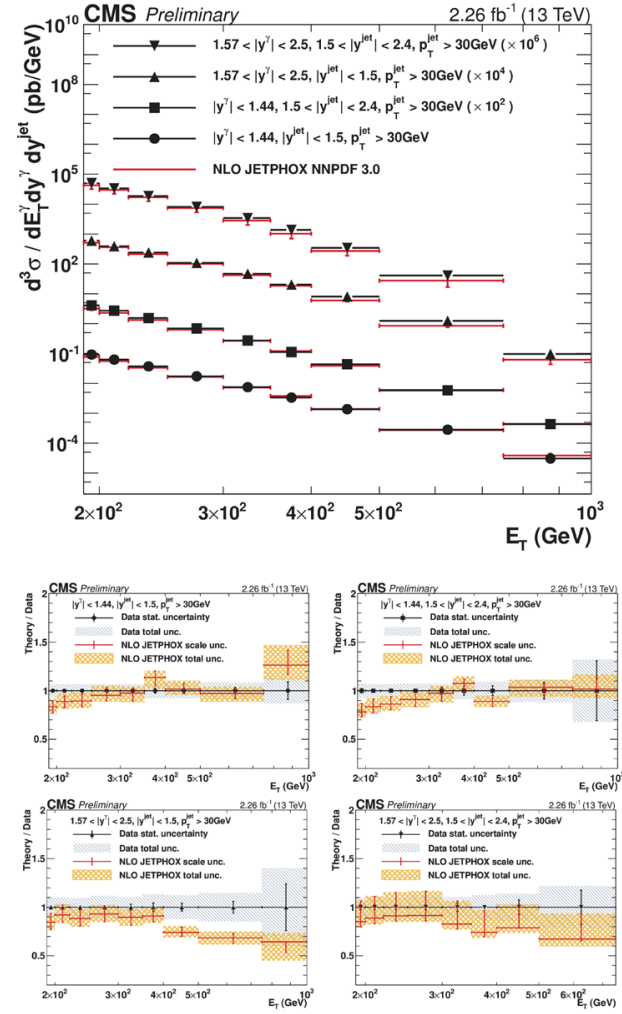

Figure 5. Upper plot: Triple-differential cross section of the production of photon in association with jets in four combinations of rapidities of photon and the highest- $p_{T}$ jet, as a function of photon $E_{T}$. Lower plot: Theory/Data plots for these rapidity bins.
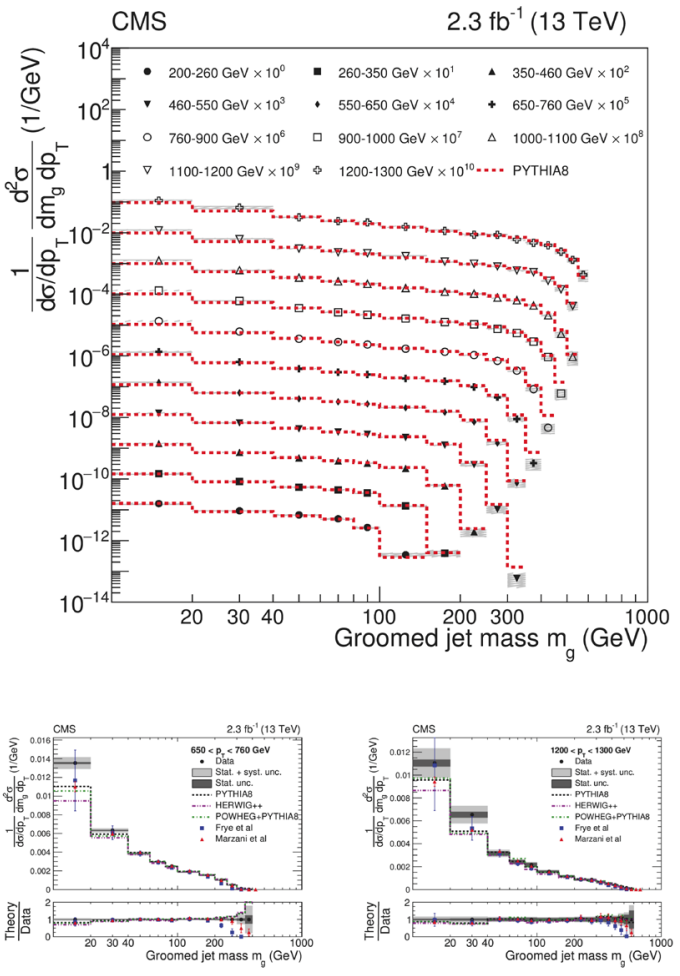

Figure 6. Upper plot: Normalized doubledifferential inclusive dijet cross section as a function of groomed jet mass for $11 p_{T}$ bins. Lower plot: Comparison of groomed jet mass with generators and theoretical calculation in two $p_{T}$ bins.

and denominator quantities. The analysis is done in four average leading-two-jet $p_{T}$ bins, resulting in measurement of $a_{S}$ for four values of energy scale $Q$, as shown in Fig. 4.

The production of a photon in association with jets is an excellent test of QCD. Inclusivephoton double-differential and photon+jet triple-differential measurements (shown in Fig. 5) with 2.26- $\mathrm{fb}^{-1}, 13-\mathrm{TeV}$ data agree well with the NLO-in-QCD JеTPнох generator [8].

The same luminosity was used for the measurement of the inclusive dijet cross section as a function of groomed and ungroomed jet mass, for $11 p_{T}$ bins of $R=0.8$ jets, as a test of soft QCD effects [9]. Good agreement with PyтнIA8 is observed, especially for groomed masses below $200 \mathrm{GeV}$, and comparisons with theoretical calculations are made, as shown in Fig. 6.

The measurement of the jet charge, defined as the sum of jet-constituent charge with three different variations of momentum weights, has been measured in $8 \mathrm{TeV}$ dijet data that correspond to luminosity of $19.7 \mathrm{fb}^{-1}$ [10].

\section{Top physics}

A $13-\mathrm{TeV}, 35.8-\mathrm{fb}^{-1}$ analysis looks for $t \bar{t}$ events in final states with lepton+jets [11]. Along with the lepton, at least 4 jets are required, at least two of which should be $b$-tagged. The 


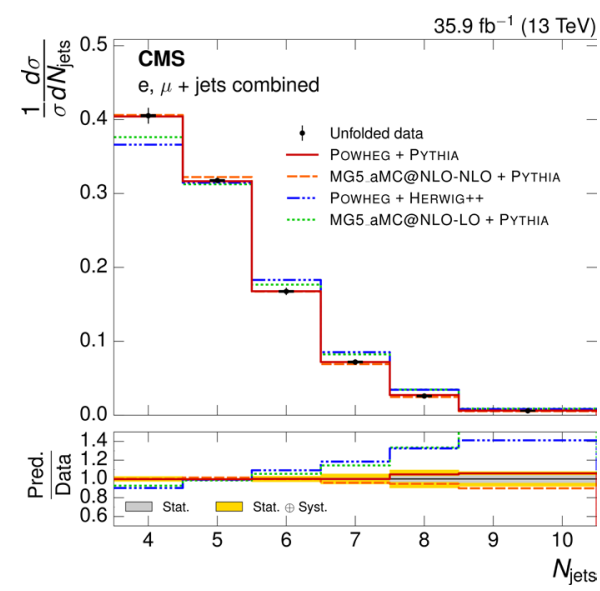

Figure 7. Jet multiplicity in top pair events in lepton+jets final states.

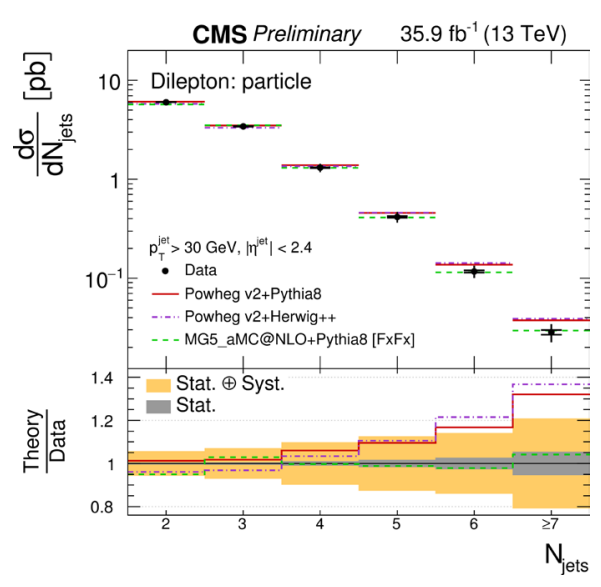

Figure 8. Jet multiplicity in top pair events in dilepton final states.

presence of extra jets is studied as a QCD test and for the determination of backgrounds in new-physics searches. Main background comes from QCD multijet events, bosons+jets and other $t \bar{t}$ channels. Powheg, with Pythia8 showering and hadronization, describes the jet multiplicity, as shown in Fig. 7, but some kinematics are only consistent with prediction after the inclusion of theoretical uncertainties in the unfolding.

The corresponding decays of the top pair to dileptons were also recently analyzed with the same luminosity [12] by requiring two leptons and at least two jets, one of which $b$-tagged. The analysis was performed in separate lepton-flavor channels which were eventually combined. Main backgrounds come from top $+W, W / Z+$ jet, diboson and $t \bar{t}+W$. Good agreement in jet-multiplicities compared to Powheg+Pythia 8 were observed, as shown in Fig. 8, and the top chromomagnetic dipole moment and its y-charge asymmetry were determined.

A recently released analysis measures the single-top production cross section in association with a photon, for muonic top decays [13], in $13-\mathrm{TeV}, 35.9-\mathrm{fb}^{-1}$ data. A muon, photon, missing transverse momentum and at least two jets are required. Main background is $t \bar{t}+\gamma$. The result is $\sigma(t \gamma+$ jets $) \times \mathcal{B}(t \rightarrow \mu v b)=115 \pm 17$ (stat) \pm 30 (syst) fb, whereas the SM prediction is $81 \pm 4 \mathrm{fb}$, which translates to a $4.4-\sigma$ evidence.

\section{Higgs physics}

The current CMS combined RunII measured Higgs boson cross section divided by the SM prediction (signal strength $\mu$ ) is $1.17 \pm 0.1$ [14]. Improvements in the precision of the $g g H$ production rate of around 50\%, compared with RunI, has been achieved due to higher luminosity and collider energy. Fig. 9 shows the good agreement of measured cross section with SM prediction for all Higgs production and decay channels.

The combination of 13-TeV data with the 7- and 8-TeV data allowed for the first observation of the Higgs boson production in association with a top-quark pair [15]. This is a 5.2- $\sigma$ discovery (expected 4.2- $\sigma$ ). All Higgs-decay channels signal strength agree with expectation, as shown in Fig. 10.

Latest results include the decay $H \rightarrow Z Z \rightarrow \ell \ell \ell \ell$ [16]. The analysis was performed separately in the $4 e, 4 \mu$, eе $\mu \mu$ channels and then combined. The data collected in 2017 were analyzed separately and combined with 2016 data for a total integrated luminosity of 77.4 $\mathrm{fb}^{-1}$. The resulted signal strength is $\mu=1.10_{-0.17}^{+0.19}$. 


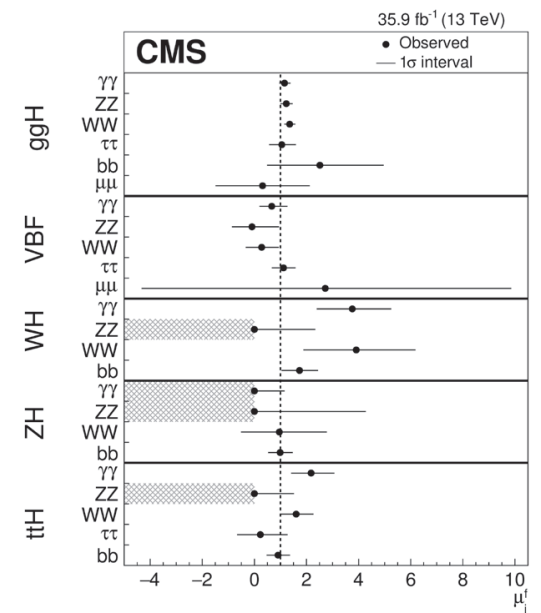

Figure 9. The current status of signal strength $\mu$ for all Higgs production and decay combinations at $13 \mathrm{TeV}$.

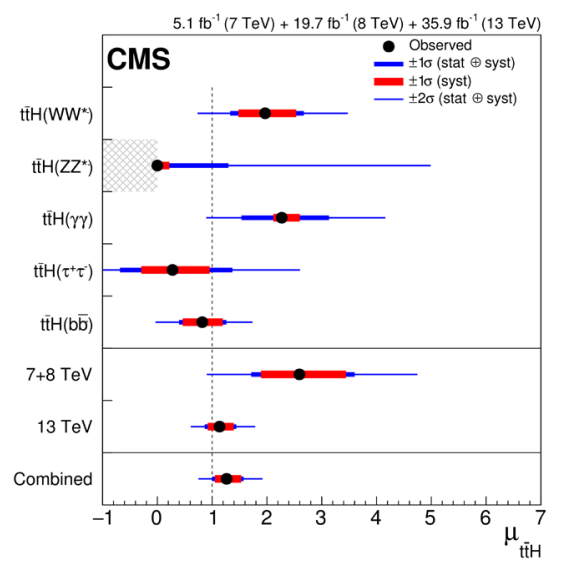

Figure 10. Signal strength for the different Higgs decay channels used in the $H t \bar{t}$ discovery.

The production of Higgs in association with a vector boson and the subsequent Higgs decay to two tau leptons was recently completed, at $13 \mathrm{TeV}$ with $35.9 \mathrm{fb}^{-1}$ [17]. At least one of the tau hadrons is required to decay hadronically. A 2.3-sigma excess in the signal strength was observed $\left(\mu=2.54_{-1.26}^{+1.35}\right)$. The results are combined with earlier $H \rightarrow \tau \tau$ analyses.

\section{Heavy-ion physics}

The CMS maintains a very active and productive heavy-ion collisions program. One of the latest released results measures the center-of-mass pseudorapidity distribution of the muon from $W^{ \pm}$boson decays in $8.16-\mathrm{TeV} p \mathrm{~Pb}$ collisions [18]. This analysis probes the nuclear PDFs of $u$ and $d$ quarks. A shown in Fig. 11, modified PDFs are needed to describe the pseudorapidity distributions.

A recent study of anisotropic flow in XeXe collisions at $5.44 \mathrm{TeV}$ and comparison with the respective $\mathrm{PbPb}$ results probes the system-size dependence and the role of initial state effects [19].

\section{Forward and small- $x$ QCD physics}

Forward and small- $x$ analyses offer valuable insight into a different range of parton dynamics. A 5- $\mathrm{TeV} \mathrm{PbPb}$ search for "light-by-light" scatter [20] led to a 4.1- $\sigma$ evidence with $\sigma(\gamma \gamma \rightarrow$ $\gamma \gamma)=120 \pm 46$ (stat) \pm 28 (syst) \pm 4 (theory) $\mathrm{nb}$. The powerful $\gamma \gamma$ acoplanarity variable was used for the separation of $\gamma \gamma \rightarrow \gamma \gamma$ signal from $\gamma \gamma \rightarrow e e$ and $g g \rightarrow \gamma \gamma$ events, as shown in Fig. 12.

\section{B physics}

Bottomonia help us study perturbation and lattice calculations of non-relativistic potentials. An analysis of $13-\mathrm{TeV}$ data using $80 \mathrm{fb}^{-1}$ of luminosity [21] led to the first observation of the resolved $\chi_{b 1}(3 P)$ and $\chi_{b 2}(3 P)$ resonances. Decays to $(Y(3 S) \rightarrow \mu \mu)(\gamma \rightarrow e e)$ were 

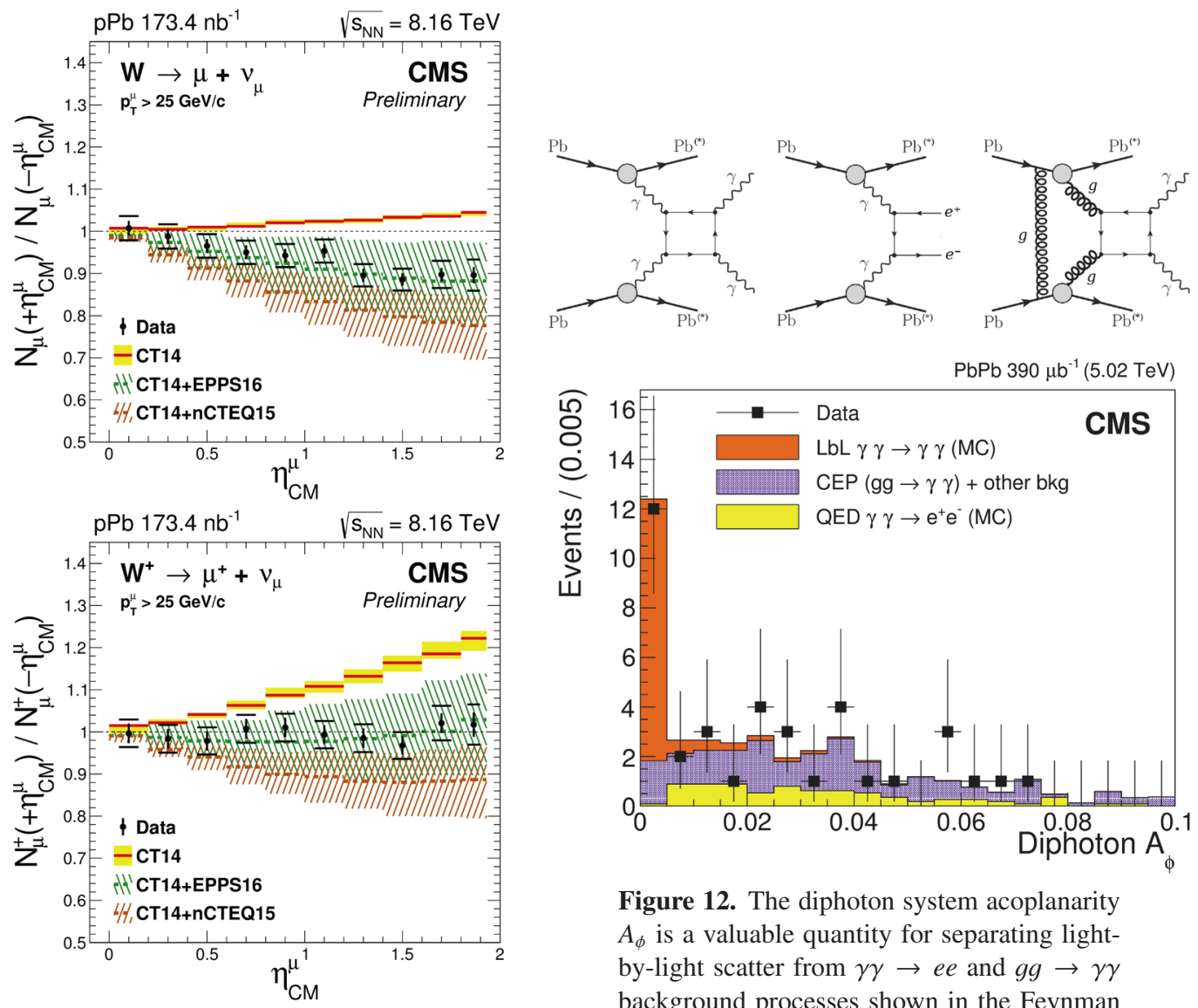

Figure 11. The forward-backward pseudorapidity ratios of the muons from the production of $W^{-}$(upper) and $W+$ (lower) bosons in $p \mathrm{~Pb}$ collisions can be explained only with nuclear modifications in the PDFs.

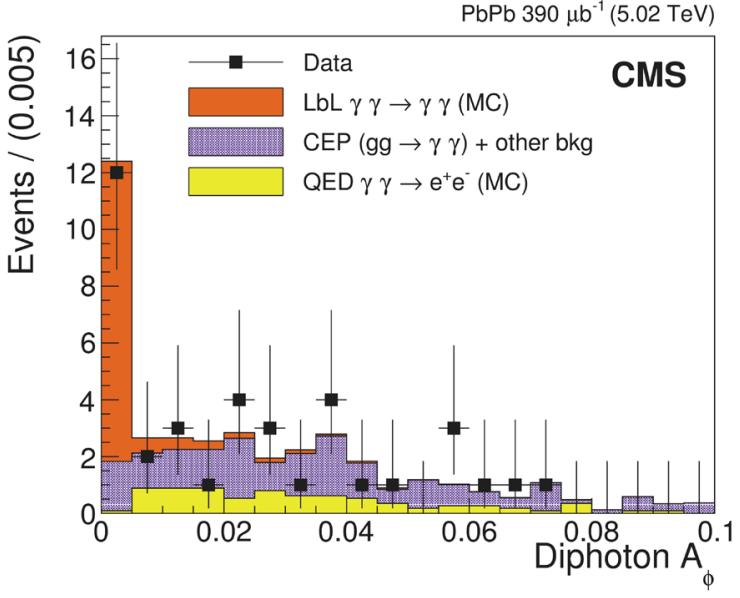

Figure 12. The diphoton system acoplanarity $A_{\phi}$ is a valuable quantity for separating lightby-light scatter from $\gamma \gamma \rightarrow e e$ and $g g \rightarrow \gamma \gamma$ background processes shown in the Feynman diagrams.

considered. The separation was achieved after a photon-energy scale calibration that utilizes $\chi_{b}(n P)$ decays to $Y(n S) \gamma$ leading to a measured $Y(3 S) \gamma$ invariant mass resolution of $2.2 \mathrm{MeV}$, as shown in Fig. 13(a). The invariant mass is shown in Fig. 13(b).

The first observation of the rare decay $Z \rightarrow J / \psi+\ell^{+} \ell^{-}$was performed with $35.9 \mathrm{fb}^{-1}$ of 13-TeV data [22]. Both direct production of $J / \psi$ and through $\psi(2 S) \rightarrow J / \psi+X$ were considered. The signal is extracted from an unbinned log-likelihood fit, resulting to $13 \pm$ 3.9(stat) $\mu^{+} \mu^{-} \mu^{+} \mu^{-}$events and 11.2 \pm 3.4 (stat) $e^{+} e^{-} \mu^{+} \mu^{-}$events leading to the measured ratio of branching fractions $\mathcal{B}\left(Z \rightarrow J / \psi \ell^{+} \ell^{-}\right) / \mathcal{B}\left(Z \rightarrow \mu^{+} \mu^{-} \mu^{+} \mu^{-}\right)=0.67 \pm 0.18$ (stat) \pm 0.05 (syst).

\section{Standard Model overview}

An overview of the measurement of production cross sections of SM processes with a great variety of final-state objects [23] shows good agreement with predictions for 7-, 8-, and 13$\mathrm{TeV}$ data. 

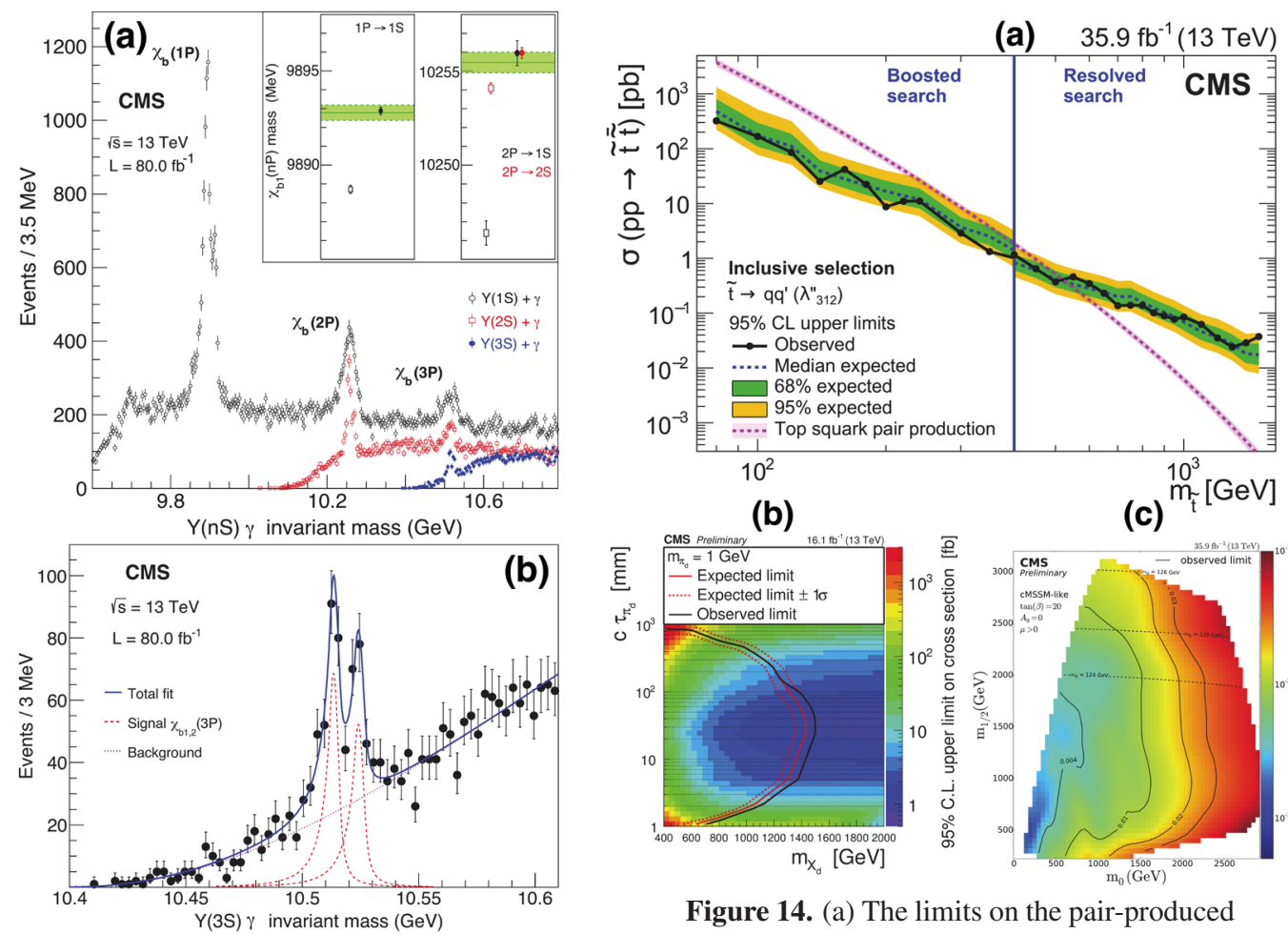

(c)

Figure 14. (a) The limits on the pair-produced scalar top decaying to diquark resonances. (b) The limit on dark quarks assuming dark pion mass of $1 \mathrm{GeV}$. (c) The $m_{1 / 2}$ vs $m_{0}$ limits of the same-sign dimuon analysis.

Figure 13. (a) The inset shows the $\chi_{b 1}(1 P)$ photon energy calibration. (b) The resolved $\chi_{b 1}(3 P)$ and $\chi_{b 2}(3 P)$ resonances.

\section{Exotic and supersymmetry physics}

CMS runs a diverse program of analyses dedicated to direct discovery of new physics, either model-independent or described by existing supersymmetric (SUSY) or exotic models.

A $13-\mathrm{TeV}, 35.9-\mathrm{fb}^{-1}$ analysis looks for pair-produced resonances decaying to diquark pairs [24]. The four jets are either reconstructed as boosted dijets, with use of appropriate grooming, for resonances below $400 \mathrm{GeV}$ or they are fully resolved above $400 \mathrm{GeV}$. A version of the analysis also requires two of the jets to be $b$-tagged. The results are consistent with expectation and limits are set on R-parity violating SUSY production pair of scalar top quarks. Fig. 14(a) shows the limit for the sub-analysis that does not require $b$-tagged jets.

Using multijet events in $13-\mathrm{TeV}, 38.5-\mathrm{fb}^{-1}$ data , an analysis searched for potential production and decay of long-lived particles through the detection of displaced vertices in the tracker [25]. The results are interpreted in R-parity violating SUSY as pair production of pairs of scalar-top quarks or gluinos or neutralinos. Cross section upper limits are set as a function of the mass and lifetime of the particles. For masses between 800 and $2600 \mathrm{GeV}$ and mean decay lengths between 1 and $40 \mathrm{~mm}$, cross sections above $0.3 \mathrm{fb}$ are excluded at 95\% confidence level.

A search for heavy, narrow resonances decaying to a Higgs boson and a photon has been completed with $13-\mathrm{TeV}, 35.9-\mathrm{fb}^{-1}$ data [26]. Investigating masses above $700 \mathrm{GeV}$ leads to boosted Higgs bosons which decay to $b \bar{b}$ pairs reconstructed as single "fat" jet. Anti- $k_{t}$ jets 
with $R=0.8$ are used with soft-drop grooming and dedicated $b$-tagging. The results agree with the SM and confidence limits are set.

A $13-\mathrm{TeV}, 16.1-\mathrm{fb}^{-1}$ analysis [27] looks for dark quarks, which are the decay products of heavy mediators in the BSSW dark-QCD model. The signature consists of 4 high- $p_{T}$ jets two from ordinary quarks and two from dark quarks, which result in emerging jets with many displaced vertices from the decay of dark hadrons to the SM charged particles. Mediator particles with masses between 400 and $1250 \mathrm{GeV}$ are excluded for dark hadron decay lengths between 5 and $225 \mathrm{~mm}$. Fig. 14(b) shows exclusion limits for models with a 1-GeV dark pion.

A 13-TeV, 35.9- $\mathrm{fb}^{-1}$ analysis looks for same-sign dimuons with at least two jets [28]. Results are consistent with SM expectation and limits are set in R-parity-violating single chargino or neutralino production in association with a muon, as shown in Fig. 14(c).

\section{Conclusions}

An overview of some of the latest and legacy CMS physics results were presented. A complete collection of CMS publications and preliminary public results can be found in https://cms-results.web.cern.ch/cms-results/public-results/publications/.

\section{References}

[1] CMS Collaboration, J. High Energy Phys. 03 (2017) 156.

[2] CMS Collaboration, Eur. Phys. J. C 76 (2016) 451.

[3] CMS Collaboration, 2017 JINST 12 P10003.

[4] M. Cacciari, G. P. Salam, and G. Soyez, J. High Energy Phys. 04 (2008) 063.

[5] CMS Collaboration, Eur. Phys. J. C 78 (2018) 566.

[6] CMS Collaboration, Eur. Phys. J. C 77 (2017) 746.

[7] CMS Collaboration, CMS-PAS-SMP-16-008 (2017).

[8] CMS Collaboration, arXiv:1807.00782, submitted to Eur. Phys. J. C (2018).

[9] CMS Collaboration, arXiv:1807.05974, submitted to J. High Energy Phys. (2018).

[10] CMS Collaboration, J. High Energy Phys. 10 (2017) 131.

[11] CMS Collaboration, J. High Energy Phys. 06 (2018) 002.

[12] CMS Collaboration, CMS-PAS-TOP-17-014 (2018).

[13] CMS Collaboration, arXiv:1808.02913, submitted to Phys. Rev. Lett. (2018).

[14] CMS Collaboration, arXiv:1809.10733, submitted to Eur. Phys. J. C (2018).

[15] CMS Collaboration, Phys. Rev. Lett. 120 (2018) 231801.

[16] CMS Collaboration, CMS-PAS-HIG-18-001 (2018).

[17] CMS Collaboration, arXiv:1809.03590, submitted to J. High Energy Phys. (2018).

[18] CMS Collaboration, CMS-PAS-HIN-17-007 (2018).

[19] CMS Collaboration, CMS-PAS-HIN-18-001 (2018).

[20] CMS Collaboration, arXiv:1810.04602, submitted to Phys. Lett. B (2018).

[21] CMS Collaboration, Phys. Rev. Lett. 121, 092002 (2018).

[22] CMS Collaboration, Phys. Rev. Lett. 121, 141801 (2018).

[23] CMS Collaboration, https://twiki.cern.ch/twiki/bin/view/CMSPublic/PhysicsResultsCombined/.

[24] CMS Collaboration, arXiv:1808.03124, submitted to Phys. Rev. D (2018).

[25] CMS Collaboration, arXiv:1808.03078, accepted by Phys. Rev. D (2018).

[26] CMS Collaboration, CMS-EXO-17-019 (2018).

[27] CMS Collaboration, CMS-PAS-EXO-18-001 (2018).

[28] CMS Collaboration, CMS-PAS-SUS-17-008 (2018). 